\title{
Design and Realization of Precise Indoor Localization Mechanism for Wi-Fi Devices
}

\author{
Weideng Su${ }^{1}$, Erwu Liu', Anna Calveras Augée ${ }^{1}$ Eduard Garcia-Villegas ${ }^{2}$, \\ Rui Wang', Jiayi You ${ }^{1}$ \\ ${ }^{1}$ School of Electronics and Information, Tongji University, Shanghai, China \\ ${ }^{2}$ Wireless Network Group, Department of Telematics Engineering, Universitat Politècnica de Catalunya, \\ Barcelona, Spain \\ [E-mail: weidengsu@tongji.edu.cn, erwu.liu@ieee.org, anna.calveras@entel.upc.edu, \\ eduardg@entel.upc.edu, ruiwang@tongji.edu.cn, youjiayi666@tongji.edu.cn] \\ *Corresponding author: Erwu Liu
}

Received July 31, 2016; revised October 13, 2016; accepted October 30, 2016;

published December 31, 2016

\begin{abstract}
Despite the abundant literature in the field, there is still the need to find a time-efficient, highly accurate, easy to deploy and robust localization algorithm for real use. The algorithm only involves minimal human intervention. We propose an enhanced Received Signal Strength Indicator (RSSI) based positioning algorithm for Wi-Fi capable devices, called the Dynamic Weighted Evolution for Location Tracking (DWELT). Due to the multiple phenomena affecting the propagation of radio signals, RSSI measurements show fluctuations that hinder the utilization of straightforward positioning mechanisms from widely known propagation loss models. Instead, DWELT uses data processing of raw RSSI values and applies a weighted posterior-probabilistic evolution for quick convergence of localization and tracking. In this paper, we present the first implementation of DWELT, intended for 1D location (applicable to tunnels or corridors), and the first step towards a more generic implementation. Simulations and experiments show an accuracy of $1 m$ in more than $81 \%$ of the cases, and less than $2 m$ in the $95 \%$.
\end{abstract}

Keywords: Wi-Fi, RSSI, Particle filters, Position measurement 


\section{Introduction}

The capability of portable devices (e.g. cellphones, smartwatches, tablets, etc.) to provide location information enables a plethora of new services and applications, such as in-building navigation, traffic control, user/machine tracking, etc. Undoubtedly, these services and applications offer numerous benefits for consumers, retailers, enterprises, and service providers. The ability to produce accurate location information of moving objects is, however, difficult to achieve, especially indoors. Lacking GPS coverage, $\mathrm{Wi}-\mathrm{Fi}$ and other wireless communications technologies have become the basis of many indoor positioning mechanisms [1]. Furthermore, at the end of 2014, near 10 billion Wi-Fi devices had been sold worldwide and about 4.5 billion Wi-Fi products are in use today [2], it is expected to surpass 15 billion by the end of 2016. With an installed base of more than 6.8 billion devices and more than 50 million public hotspots worldwide, Wi-Fi has become almost ubiquitous. Said ubiquity shortens and simplifies the development of new location-based applications using Wi-Fi, as compared to other technologies. During the last decade, considerable research was conducted on Wi-Fi-based positioning, but the poor accuracy typically obtained was clearly unsatisfactory for many use cases. In this sense, the IEEE P802.11 TGaz ${ }^{1}$ is currently studying new amendments to enhance accuracy and scalability of positioning for Wi-Fi equipment. Aligned with those objectives, in this paper we present Dynamic Weighted Evolution for Location Tracking (DWELT); an enhanced mechanism intended to provide accurate positioning of Wi-Fi devices, leveraging the ubiquity of Wi-Fi infrastructure in many scenarios. As shown throughout this paper, DWELT is simple, fast, and accurate.

Different Wi-Fi-based positioning schemes exist in the literature [3]. Angle of Arrival (AoA) techniques determine the direction of propagation of the node's transmission incident on an antenna array by measuring the Time Difference of Arrival (TDoA) at each antenna. TDoA itself and other Time of Flight (ToF) solutions measure the time it takes for a radio signal to travel from a transmitter to a distant receiver antenna. Based on that, the distance can be directly calculated from those samples as signals travel at a known speed. Alternatively, RSSI-based approaches, such as fingerprinting or signal propagation model (SPM) schemes are based on the measurement of received signal power. In general, AoA and ToF techniques ask for complex hardware, or strict time synchronization, which is impractical in many applications. RSSI-based schemes do not require any special or sophisticated hardware and, therefore, can be easily implemented given that most off-the-shelf Wi-Fi devices report RSSI from in-range APs, and commercial APs can easily keep track of the power received from multiple client stations.

Fingerprint-based algorithms include two phases: an off-line training phase and the on-line pattern-matching phase. During the first phase, the area of interest is thoroughly surveyed to build a database with as many digital fingerprints as possible (e.g. number of Wi-Fi APs and the power received from each of those APs at every possible location). However, this off-line training is costly [4] and needs to be repeated frequently to

\footnotetext{
${ }^{1}$ http://www.ieee802.org/11/Reports/tgaz_update.htm
} 
accommodate changes in the environment (replacement of APs, changes in the structure of the building or even changes in the furniture, etc.). In order to speed up this phase, Tang et al. [5] propose to use unmanned ground vehicles while, in [6], authors use customized Wi-Fi APs along with Wi-Fi-based sensor anchors strategically placed to automatically calibrate and update the RSSI database. Mo et al. [7] propose a localization technique that is able to build and update radio fingerprints. Besides, Huang et al. [8] and Bruno et al. [9] have well studied the Simultaneous Localization and Mapping (SLAM) scheme based on Wi-Fi devices to reduce runtime complexity and improve location accuracy. In addition, Wang et al. [10] propose a framework for unsupervised indoor localization called UnLoc, and try to achieve the tradeoff between accuracy and calibration overhead. Furthermore, Lim et al. [11] build an indoor Wi-Fi localization system with smart antennas, which avoids the use of the off-line training phase, to triangulate the target.

Unlike many fingerprint methods, where the target's location is derived from the measurements provided by all available sensors, in [12], the Fuzzy C-Means clustering algorithm is utilized to focus only on a certain cluster of fingerprints, achieving a time-efficient localization with good resolution. A similar approach is proposed in [13], where the authors adopt a K-means clustering to compare measured RSSI values for a coarse positioning and adopt Genetic Algorithm to improve precision. Moreover, Shon et al. [14], propose a cluster-based multidimensional scaling (MDS) for range-free localization and yielded a significant accuracy improvement. Gogolak et al. [15] take advantage of the learning of the neural network model based on preprocessed (mean, median, standard deviation) RSSI values, while the system is trained with mean RSSI values. More recent works combine RSSI measurements with other data available at the target device (e.g. built-in compass, motion sensors, etc. in smartphones or other wearable devices [16], [17]).

On the other hand, SPM-based methods apply a propagation path loss model to derive the distance of a transmitter from the power received. However, received power can be highly variable in a dynamic scenario, since radio signals undergo many physical phenomena as they propagate through obstacles or reflect off static (or moving) objects. All these phenomena introduce noise in power measurements, which deviate from the predictions of the propagation models. In response to the fluctuation of the determined RSSI values, the theory of maximum entropy is introduced to eliminate the Gaussian noise in [18]. Besides, Huang et al. [19] develop a real-time RFID indoor positioning based on Kalman-filter to mitigate the drift of RSSI values. It is worth mentioning that Solhjoo and Tinati [20] try to find out the relationship between RSSI values and signal propagation distance among different direction of APs, and propose a non-circular signal propagation model. In [21], Guo et al. develop an Exponential-Rayleigh (ER) model, which includes two parts, the large-scale part and the small-scale part, to mitigate the interferences caused by the multi-path when the signal is propagating. Similarly, in [22], Lim et al. investigate a Dual Log Path Loss Model that includes two Log-curves (one is for the distances under $5 \mathrm{~m}$, and the other for distances between $5 \mathrm{~m}$ and $10 \mathrm{~m}$ ).

DWELT is an SPM-based scheme, which includes an adaptive propagation loss model 
and a particle-filtering algorithm; both mechanisms intend to overcome the intrinsic limitations of SPM schemes.

\section{Description of DWELT}

In this paper, we target a quick, efficient, and accurate localization implementation, which does not require any centralized intelligence. The implementation is generally independent of the specific hardware or application situations, and needs no prior information harvesting.

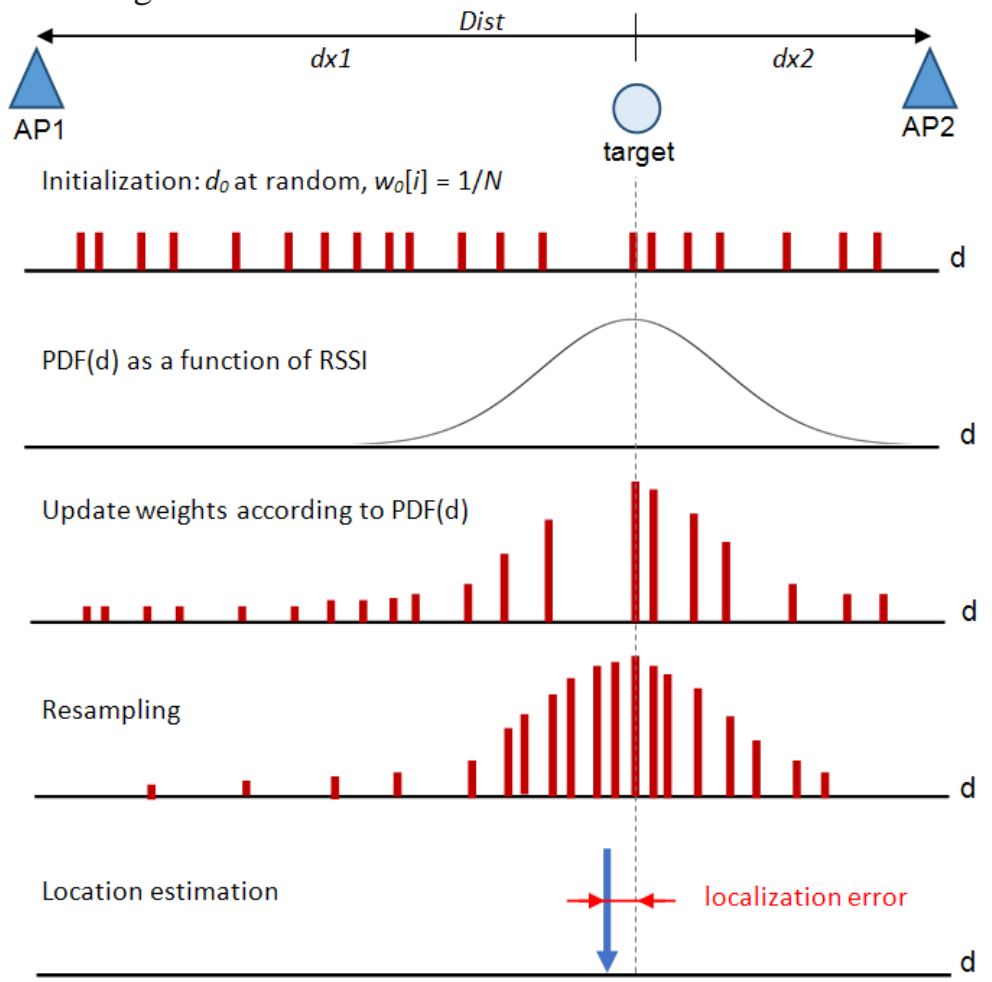

Fig. 1. Illustration of DWELT localization process (example with $N=20$ in a 1D scenario)

To ease the understanding of DWELT algorithm, we assume a 1D scenario as depicted in Fig. 1, which is similar to the Wi-Fi devices deployed in a long runnel or corridor. In this case, Wi-Fi APs and the target device are in a line. $N$ refers to the total numbers of random locations, $w_{0}[i]$ refers to the initial weight of the $i^{\text {th }}$ random location. The distance between any two adjacent APs (Dist) is known, and the target device is located between any two APs (at distance $d_{x 1}$ from AP1 and $d_{x 2}$ from AP2) ${ }^{2}$. For such

\footnotetext{
${ }^{2}$ In a real scenario, the different elements can be placed at different heights, in which case, basic trigonometric rules can be applied to compensate for the possible loss of precision.
} 
scenario DWELT models the localization problem as a stochastic process in which the estimation of location is represented as probability distributions. More importantly, DWELT applies a weighted posterior-probabilistic evolution for quick convergence of localization, as detailed in the following.

\subsection{Path loss model}

Logically, the pivotal component of an SPM-based positioning method is to accurately estimate the path loss in the selected environment. In practice, however, no analytical model exists to provide accurate predictions for all possible environments. This is particularly true for the type of environments of our interest, where the radio waves are affected by the working frequency, the roughness and electrical conductivity of walls, geometry, etc. Most indoor propagation models are based on the breakpoint model [23] (also known as X-slope model), where the signal attenuation is characterized by a different exponent on several (X) discrete intervals from the transmitter. In our work, we assume distances of less than $100 \mathrm{~m}$ and, similar to [24], we adopt a two-slope path loss model, represented in (1), which provides an adequate accuracy at low computational cost $^{3}$.

$$
P_{d x}=P_{0}-10 \eta \log \left(\frac{d_{x}}{d_{0}}\right)+N_{\sigma}
$$

where,

$d_{0}$ refers to the breakpoint distance (in $m$ ); $d_{0}$ is a tunable parameter depending on the environment.

$d_{x}$ is the distance from the transmitter $\left(d_{x}>d_{0}\right)$.

$P_{0}$ refers to the RSSI (in $d B m$ ) measured at $d_{0}$.

$P_{d x}$ refers to the RSSI (in $d B m$ ) measured at $d_{x}$.

$\eta$ is the path loss exponent applicable after the breakpoint.

$N_{\sigma}$ represents the noise power as a Gaussian random variable with zero mean and $\sigma$ standard deviation.

Similar to [25], in order to apply the algorithm in different environments without a costly off-line training phase, DWELT performs a real-time estimation of the path loss exponent $(\eta)$ in the signal propagation model shown in (1).

\subsubsection{Estimation of path loss exponent}

For the sake of simplicity we temporarily assume noise power $\left(N_{\sigma}\right)$ to be $0 \mathrm{dBm}$,

\footnotetext{
${ }^{3}$ The propagation loss model can be sophisticated to better reflect the particularities of other scenarios (e.g. add losses due to walls/floors) without affecting other components of DWELT.
} 
breakpoint distance $\left(d_{0}\right)$ to be $1 m$ (typical choice in indoor scenarios). From equation (1) the distance $d_{x}$ from a transmitter is given by

$$
d_{x}=10^{\frac{P_{0}-P_{d x}}{10 \eta}}
$$

If $\mathrm{RSSI}_{1}$ corresponds to the average RSSI with which AP1's transmissions are measured at the target location (see Fig. 1) and $R_{S S I}$ corresponds to AP2, the distance between AP1 and AP2 (Dist) is approximated by:

$$
\text { Dist } \approx d=10^{\frac{P_{0}-R S S I_{1}}{10 \eta}}+10^{\frac{P_{0}-R S S I_{2}}{10 \eta}}
$$

Logically, $d$ should be as close to Dist as possible and, to this aim, we adjust $\eta$ from $a$ to $b$ in a step of $\Delta$. The chosen $\eta$ is the value that minimizes $|d-D i s t|$. Parameters $a, b$ and $\Delta$ are left as an implementation choice; $a$ and $b$ should cover the range of expected values for the path loss exponent of the selected environment (typically between 2 and 5 for common wireless environments) and $\Delta$ depends on the tradeoff between resolution and computation time.

\subsection{Location estimation}

With accurate estimation of the path loss exponent, distance estimations derived from RSSI are still unreliable due to the diverse phenomena affecting propagation. Those phenomena, especially in the presence of moving objects, cause RSSI measurements to vary in time (even for a fixed location) and make the RSSI vs. distance relationship differ from the expected monotonically decreasing behavior. Hence, we process raw RSSI data through a filtering algorithm, as illustrated in Fig. 1.

\subsubsection{Initialization}

First, we generate a set of $N$ random locations with uniform probabilities and store them in the array $d_{0}[N]$. The array $d_{t}[N]$ represents the set of possible distances from a given AP1 over the line between AP1 and AP2 (i.e. possible values for $d_{x 1}$ in Fig. 1), at time $t$. Then, we set a weight for each of those locations and store them in the array $w_{t}[N]$. In this initialization, all $N$ locations have the same weight of $w_{0}[i]=1 / N, i=1,2, \ldots, N$.

\subsubsection{Weight update}

Assume $v_{\max }$ is the maximum velocity of the targeted object (e.g. the walking speed of human beings in indoor environments is typically less than $2 \mathrm{~m} / \mathrm{s}$ ). The orientation of 
the object, represented by $\vec{O}$, can be either +1 (i.e., moving from AP1 towards AP2) or -1 (i.e., from AP2 to AP1). The time interval between consecutive iterations is denoted as $\delta$. At each iteration, every possible location in $d_{t}$ is updated by

$$
d_{t}[i]=d_{t-1}[i]+\delta \cdot v_{\max } \cdot \vec{o}
$$

Then, we update the corresponding weights using Eqs. (5) (6) (7), where $w_{t}[i]$ represents the probability according to a normal distribution, $d_{t}[i]$ is the actual location, given the measured RSSI (RSSI 1 and $\left.R S S I_{2}\right)$ and the expected RSSI at $d_{t}[i]$, and $w_{A P 1}$, $w_{A P 2}$ represent the portion of the weight that measures the probability of a location according to its distance from AP1 and AP2, respectively. Note that weights $w_{t}[i]$ are normalized so that $\sum_{i=1}^{N} w_{t}[i]=1$.

$$
\begin{gathered}
w_{t}[i]=w_{A P 1}+w_{A P 2} ; i=1,2, \ldots, N \\
w_{A P 1}=\frac{1}{\sigma \sqrt{2 \pi}} e^{-\frac{\left(R S I_{1}-\left(P_{0}-10 \eta \log \left(\left|d_{t}[i]-0\right|\right)\right)\right)^{2}}{2 \sigma^{2}}} \\
w_{A P 2}=\frac{1}{\sigma \sqrt{2 \pi}} e^{-\frac{\left(R S S I_{2}-\left(P_{0}-10 \eta \log \left(\left|D_{i s t}-d_{t}[i]\right|\right)\right)\right)^{2}}{2 \sigma^{2}}}
\end{gathered}
$$

\subsubsection{Resampling}

Once the array of weights has been updated, we can see that some locations stemmed from (4) are very unlikely (i.e. have a small weight, less than $\alpha$ ). Before the next iteration, we remove some of those outliers. Due to unpredictable variations of RSSI, the detection of invalid locations may not be trivial; a simple truncation selection (remove a fixed portion of the weakest candidates) may end up, for example, removing good choices that, due to a temporary fading, seemed unlikely. Instead, we follow a fitness proportional selection, where those locations with lower weight are selected for removal with higher probability; in this way, some weaker solutions may survive the selection process, which could prove useful in future iterations. The resampling process is finalized by replacing those removed locations with new random values uniformly distributed within a small area $(\theta)$ around the last estimated position, $\hat{d}_{t}$.

\subsubsection{Localization results}

After completing all the steps for a given number of iterations, we compute the weighted average of all the possible locations and provide this value as the estimated location of the targeted object at time $t$. This is shown in (8), as the dot product $w_{t} \cdot d_{t}$. 


$$
\hat{d}_{t}=\sum_{i=1}^{N} w_{t}[i] d_{t}[i]
$$

As shown in Algorithm 1, the DWELT algorithm starts at the online real-time estimation of path loss exponent, and continues to initialization process. After that, DWELT continues to update the weights of particles in accordance with up-to-date RSSI readings. In order to remove unlikely locations and not to remove good choices wrongly in case of a temporary fading, we adopt a fitness proportional selection as resample algorithm. Finally, the algorithm will decide the flow direction according to the convergence of DWELT and the maximum iterations. Note that the path loss exponent will be updated (estimated in real time) before every localization process.

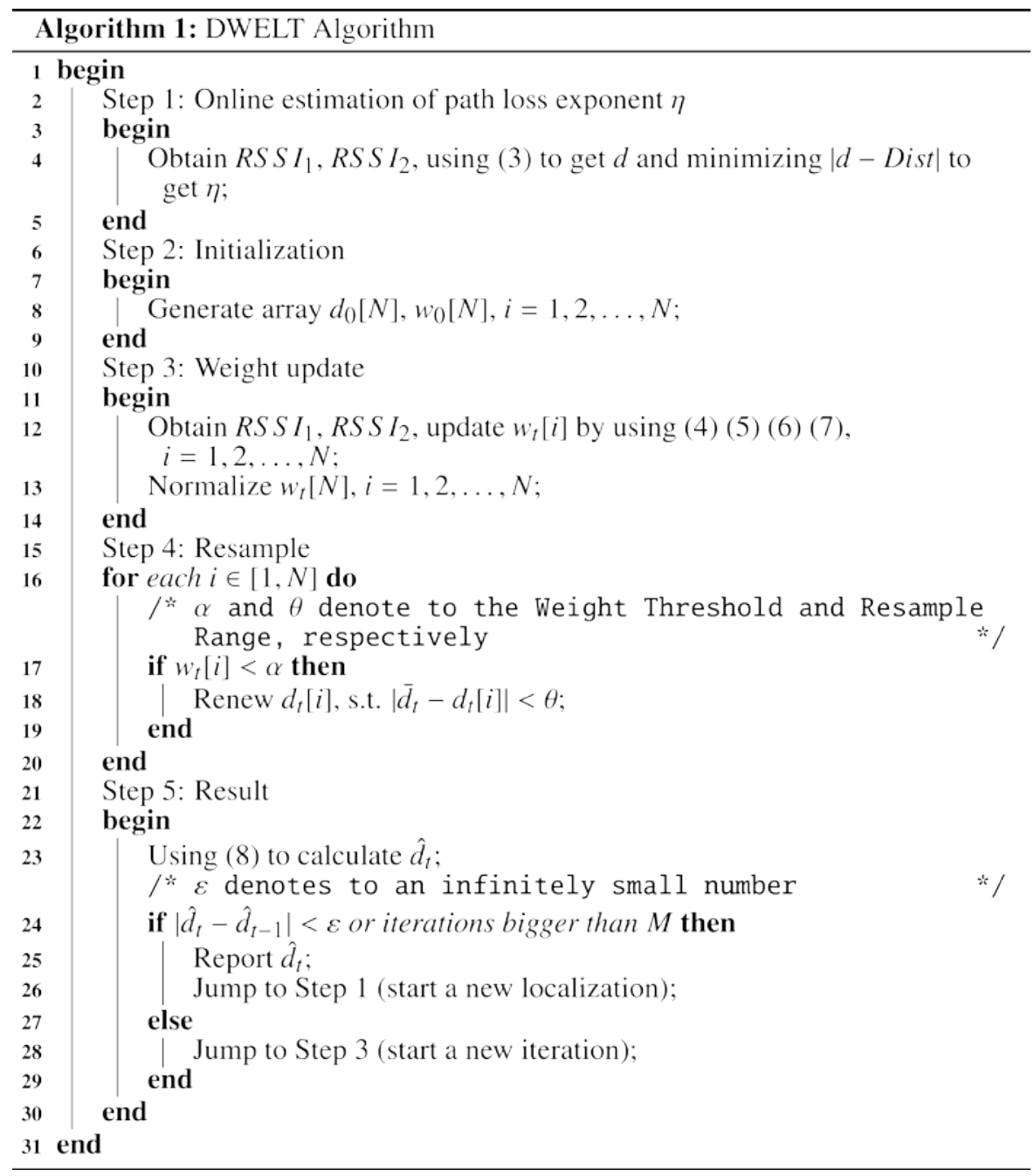




\section{Performance of DWELT}

The previous section described DWELT through an initial and simplified 1D approach. The 1D approach can be feasibly extended to provide 2D positioning scenario in more generic environments (e.g. for guided shopping, navigation in office buildings, convention centers, airports, etc.). In the 2D adaptation of DWELT, the randomly generated positions are distributed over a plane instead of a line and are then weighted according to the RSSI measured from three APs or more.

This section presents the evaluation of DWELT and the influence of different design choices, firstly, by means of Matlab simulations and, secondly, through measurements with a real testbed deployed in different small scenarios.

\subsection{Simulation results}

As depicted in Fig. 1, we assume APs are deployed every Dist over a straight line, and the exact location of each AP is known in advance. The target, which is randomly placed at the beginning of the simulation, is provisioned with noisy RSSI measurements from all surrounding APs, according to (1). Then, for a 1D scenario (APs and target device in the same line), we identify the two APs providing the strongest signals as AP1 and AP2. In a real deployment, AP1 and AP2 would be identified by their MAC address (or unique BSSID).

In the simulation, we assume a maximum pedestrian speed $v_{\max }=2 \mathrm{~m} / \mathrm{s}$ (the mean walking speed of pedestrian is around $1.34 \mathrm{~m} / \mathrm{s}$ [26]); the moving direction of every particle generates randomly (either +1 or -1 ) at every iteration; the determined RSSI value at the reference distance $d_{0}(1 \mathrm{~m})$ is $P_{0}=-35 \mathrm{dBm}$ [25] (the average of hundreds of samples); the path loss exponent $\eta$ is adapted with values from 2 to 5 [25] [27] in steps of 0.03 (a fixed value of 3.5 is used to generate RSSI samples); the distance between every two adjacent APs is Dist $=60 \mathrm{~m}$, similar to [25]; the time interval between consecutive iterations is $\delta=0.05 s$ (the computation time for one iteration loop is about 0.05 s); and the noise power is $4 d B$, similar to [27], see Table 1.

Table 1. DWELT Configuration Parameters

\begin{tabular}{|c|c|c|c|c|c|}
\hline$v_{\max }(m / s)$ & $P_{0}(d B m)$ & $\eta$ & $\operatorname{Dist}(m)$ & $\delta(s)$ & $N_{\sigma}(d B)$ \\
\hline 2 & -35 & $2 \sim 5,0.03$ & 60 & 0.05 & 4 \\
\hline
\end{tabular}

In order to show the convergence of the proposed algorithm, the simulation result is demonstrated in Fig. 2. And the vertical coordinate of this figure represents the distance between the target and AP1. As we can see from Fig. 2, the positioning precision for 50 iterations (Platform: Matlab, CPU: $1.8 \mathrm{GHz}$, Elapsed Time: $68 \mathrm{~ms}$ ) can be $0.9 \mathrm{~m}$ stably. We can draw a conclusion that, the proposed algorithm is an evolutionary algorithm which is based on dynamic enhancement, it can track and locate the target through accurately and requires short calculation time. 
After that, we conduct more simulations to see how the number of particles $(N)$ and noise power $\left(N_{\sigma}\right)$ affect the localization results. For that, we assume the maximum number of iterations (NoI) is 50, and the other configuration parameters are the same as Table 1.

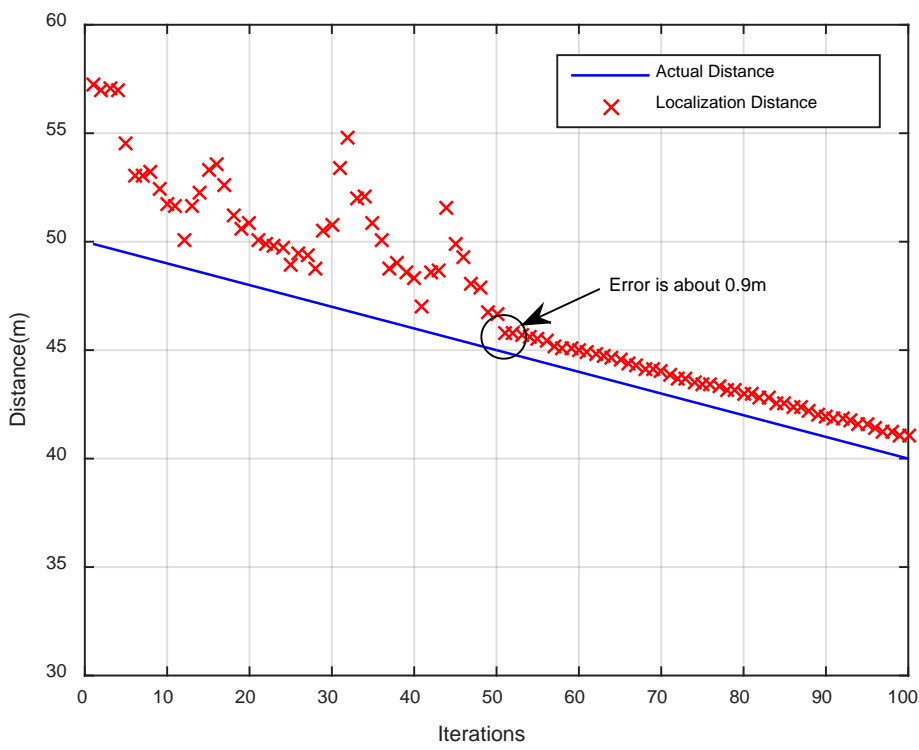

Fig. 2. Simulation and evaluation for positioning and tracking

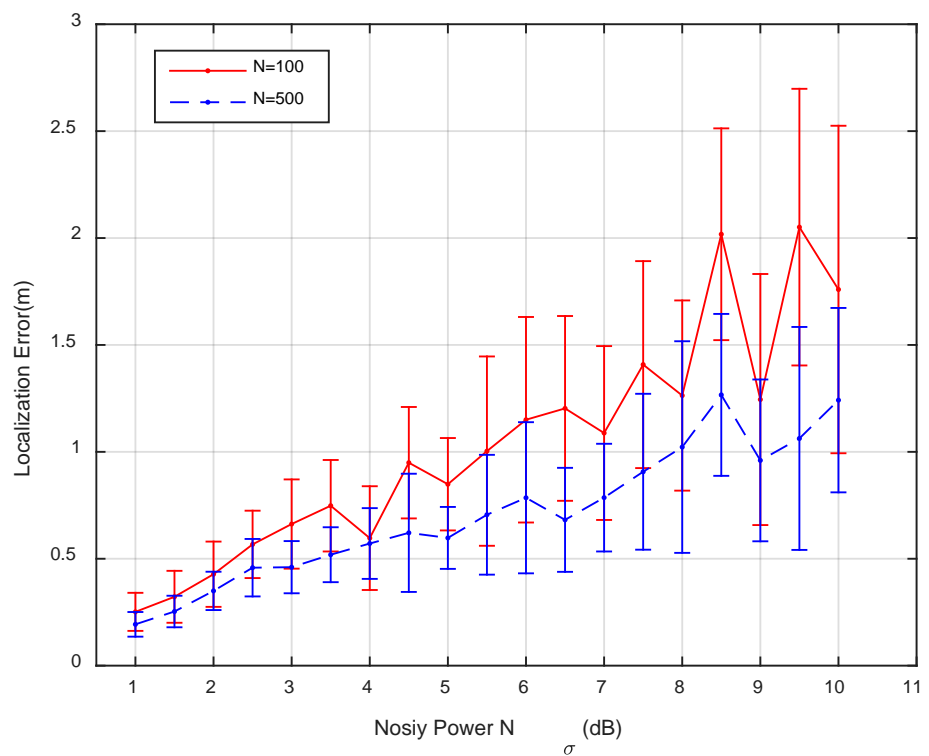

Fig. 3. Simulated localization error with noisy RSSI measurements for 100 and 500 particles 
Fig. 3 shows that the 1D resolution of DWELT varies, on average, from $20 \mathrm{~cm}$ to $2.0 \mathrm{~m}$, in the worst case. Logically, as we increase the noise in RSSI measurements, we witness a noticeable loss of precision. That loss of precision can be mitigated by increasing the number of particles $(N)$ of the filtering algorithm. In this regard, note that Fig. 3 shows localization errors for two cases: $N=500$ and $N=100$. A larger number of candidate positions provide an improved accuracy (above $1 \mathrm{~m}$, in the worst case) at the cost of an increased computation time. Note that, in typical indoor WLAN scenarios, the standard deviation of $N_{\sigma}$ is between 3 and $6 d B$ [28], resulting in $\left|N_{\sigma}\right|$ below $4 d B$ for $50 \%$ of the cases, and below $10 \mathrm{~dB}$ in $90 \%$.

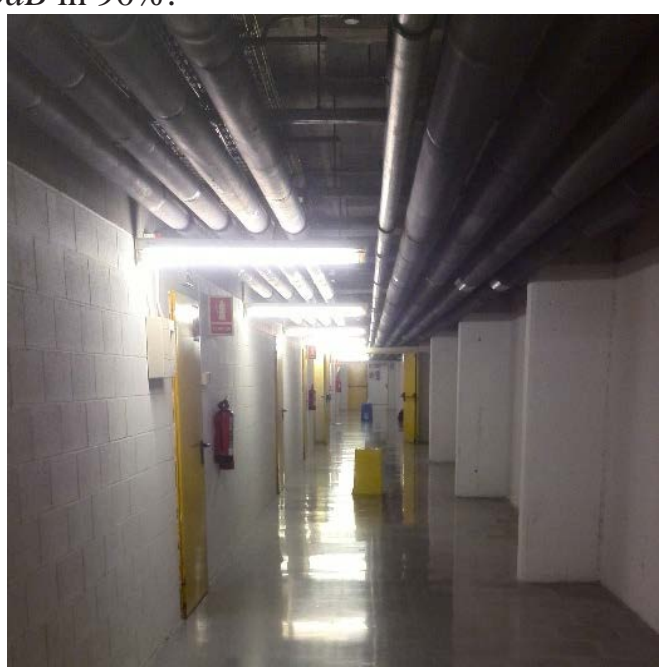

Fig. 4. Corridor environment

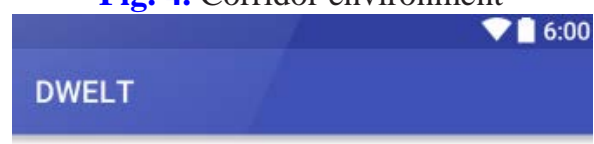

Settings:

Input SSID1 Input SSID2

Input distance between APs

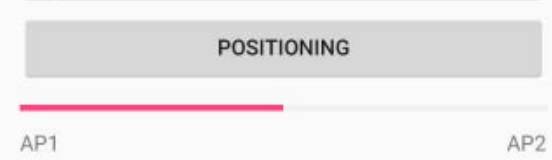

Current Location: $10.00 \mathrm{~m}$

Note: The current location shows the distance between the target and AP1

Fig. 5. User interface of DWELT APP 


\subsection{Experimental results}

We conducted several experiments in different scenarios, one of the experimental environment is depicted in Fig. 4 (corridor environment). The testbed consists of two off-the-shelf Wi-Fi APs (Aigo RS150) and a HUAWEI U9508 ${ }^{4}$, an Android-based (Jelly Bean 4.1.2) smartphone running DWELT. The configuration parameters are the same as in the simulation environment except for the distance between APs, Dist $=20 \mathrm{~m}$. The experimental setup is similar to Fig. 1, and the user interface of Android APP running DWELT algorithm is shown in Fig. $\mathbf{5}$.

To evaluate how the real-time estimation of path loss exponent affects the performance of localization algorithm, we conducted several experiments in corridor environments, shown in Fig. 6. From this figure, we notice that the real-time estimation of path loss exponent significantly improves the performance of localization algorithm, we see that about $46 \%$ improvement can be achieved compared to that without path loss exponent estimation process to achieve an accuracy of $2 m$.

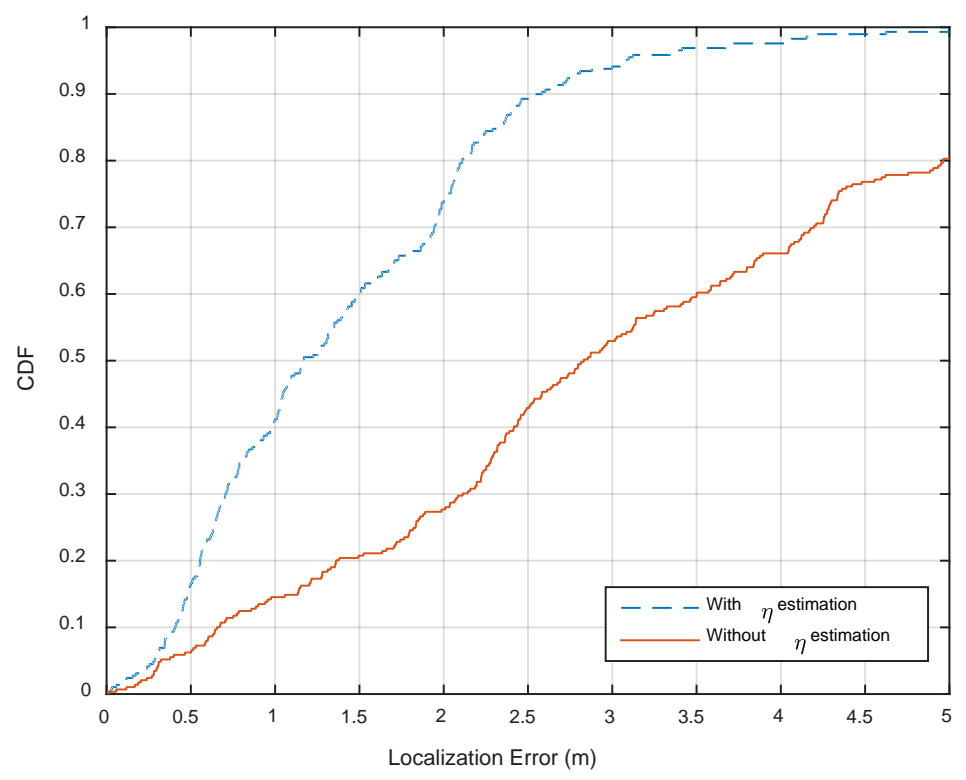

Fig. 6. CDF of localization error with/without real-time path loss exponent estimation

\subsubsection{Enhanced resample/weighted strategy}

The experiments of the first implementation show a poor precision of $1 \mathrm{~m}$ only in $42 \%$ of the cases. It is clear that the algorithm needs adjustments to bring it to practice. In this regard, the first set of experiments focused on the resampling phase. Initially, the set of

\footnotetext{
CPU.

${ }^{4}$ In this platform (Quad-core 1.4GHz CPU, 2GB of RAM), the DWELT application represents a peak of $29 \%$ of the
} 
new possible locations was generated within the whole range from 0 to Dist. Intuitively, reducing the range for new candidate locations allows more precise estimations with fewer iterations, in most of the cases. Therefore, we modified DWELT to generate new candidate locations within the region between $\hat{d}_{t}-\lambda$ and $\hat{d}_{t}+\lambda$ ( $\hat{d}_{t}$ is the most recent estimated location). Experimentally we observed that values below $\lambda=5 \mathrm{~m}$ do not show any further improvements.

Another interesting observation was that DWELT provided more accurate results when the targeted object was not in close proximity of any of the reference APs. We realized that, near the breakpoint distance, the path loss model fails to provide accurate RSSI predictions, while it is more reliable in the far-field. Accordingly, we evaluated different strategies intended to overcome that significant limitation of the model; giving more relevance to the RSSI of the farthest AP was identified as the best approach. In consequence, the weight distribution shown in (5) is slightly modified so that, when the targeted object estimated location is closer to AP1 (less than Dist / 4 ), we give $50 \%$ added weight to RSSI2, and vice versa.

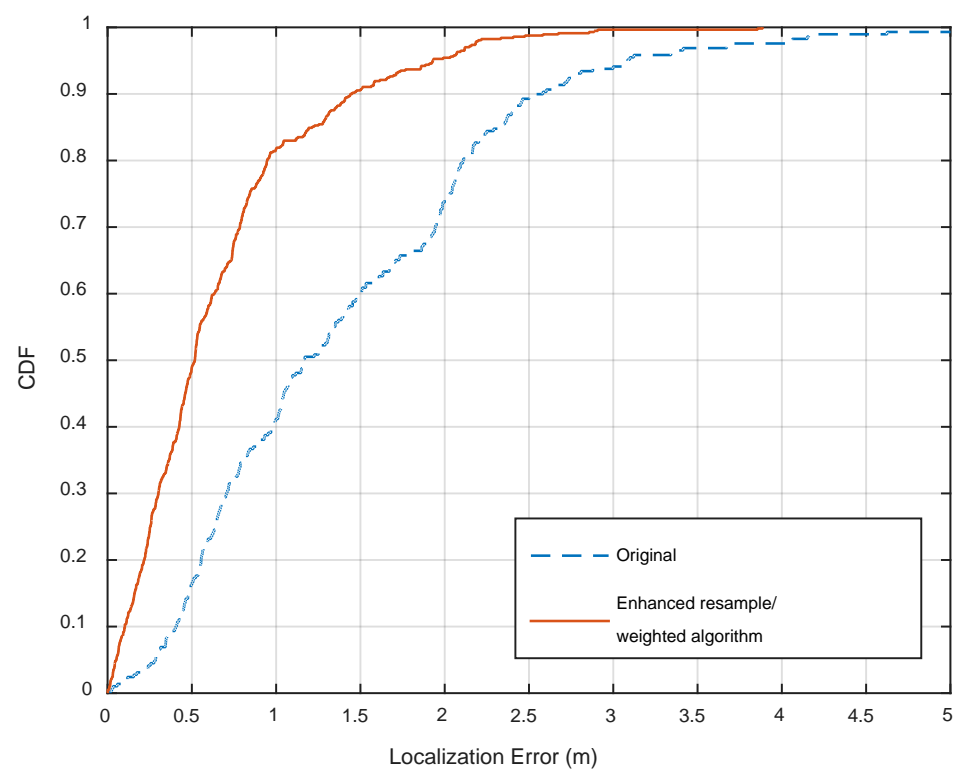

Fig. 7. CDF of localization error with/without enhanced resample/weighted algorithm

As shown in Fig.7, the localization performance is improved significantly with enhanced resample/weighted algorithm. It achieves an accuracy of $1 \mathrm{~m}$ at $81 \%$, of $2 \mathrm{~m}$ at more than $90 \%$.

After implementing the enhanced resample and weighted strategies, DWELT was tested in three different scenarios: an indoor parking lot, a crowded shopping mall and a long corridor, and the distance between APs is $20 \mathrm{~m}$. In Table 2, the average localization errors of 3 different scenarios are less than one meter, and the precision of DWELT 
shows good robust. More importantly, the average localization error and maximum error of DWELT respectively fall by $55.4 \%$ and $5.1 \%$ compared with SLAM [5] [8] [9] algorithm, see in Table 3, Fig. 8. After that, Fig. 9 shows the CDF of the localization error in those three scenarios; the $90^{\text {th }}$ percentile varies between $1.4 \mathrm{~m}$ (corridor) and $2 \mathrm{~m}$ (parking lot).

Table 2. Localization error of 3 different scenarios

\begin{tabular}{|c|c|c|c|}
\hline & Corridor & Mall & Parking lot \\
\hline The average error $(\mathrm{m})$ & 0.6656 & 0.9430 & 0.9560 \\
\hline The max error $(\mathrm{m})$ & 3.1916 & 5.0537 & 3.8862 \\
\hline
\end{tabular}

Table 3. Localization error of 2 different methods

\begin{tabular}{|c|c|c|}
\hline & SLAM & DWELT \\
\hline The average error $(\mathrm{m})$ & 1.9313 & 0.8608 \\
\hline The max error $(\mathrm{m})$ & 5.3212 & 5.0537 \\
\hline
\end{tabular}

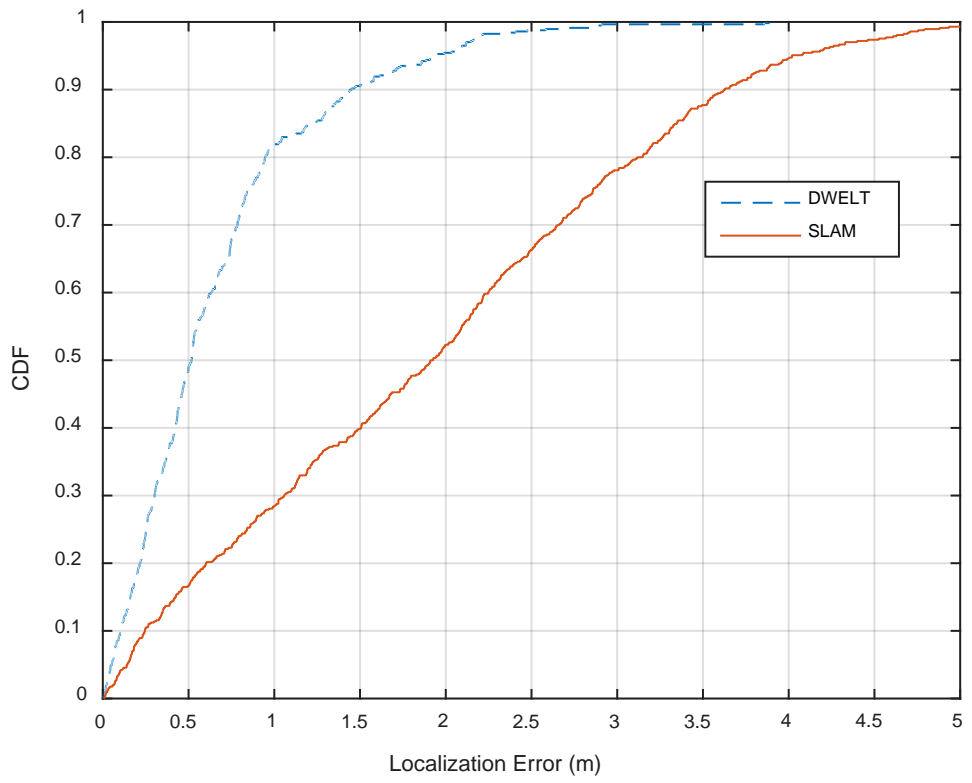

Fig. 8. CDF of 2 different localization methods 


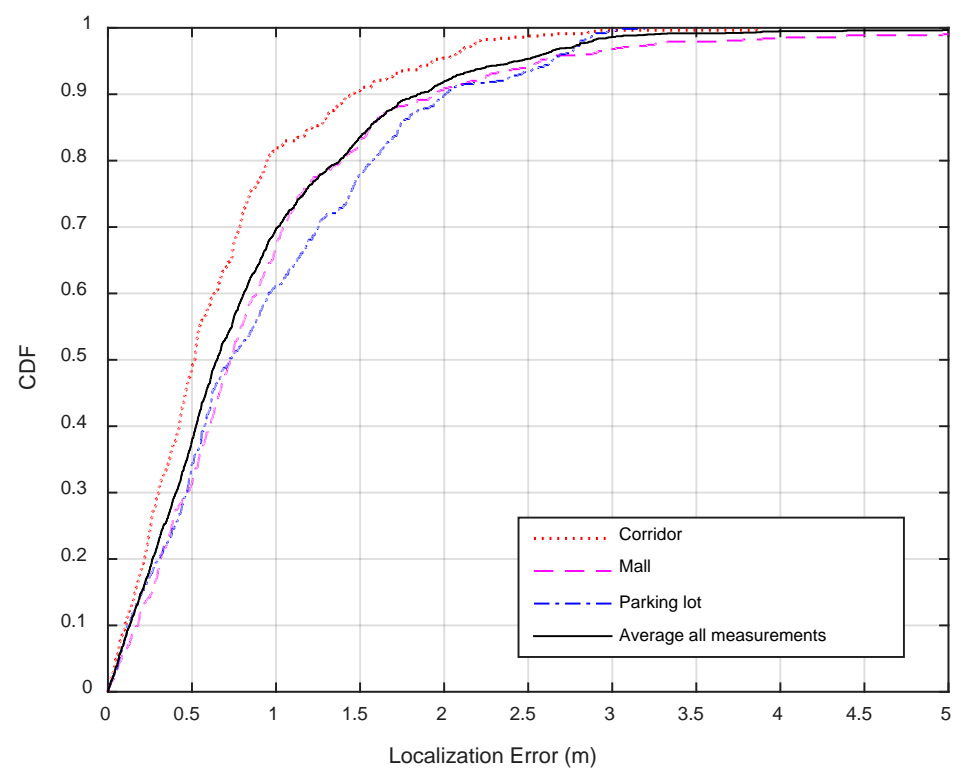

Fig. 9. CDF of the measured localization error in three different scenarios

\subsubsection{Performance vs. APs' distance}

The resolution of RSSI in Wi-Fi device (typically $1 \mathrm{~dB}$ ) restricts the maximum distance between the target object and AP in SPM-based positioning. According to equation (1), to meet $P_{d x 1}-P_{d x 2}=1 d B$, the relationship between $d_{x 1}$ and $d_{x 2}$ must satisfy $d_{x 2}=d_{x 1} \cdot 10^{1 / 30}=1.0798 d_{x 1}$, when the noise powers in two different locations are the same and the path loss exponent is 3 . Therefore, when $d_{x 1}>20 m$, it will take a distance of at least $1.5955 \mathrm{~m}\left(0.798 \cdot d_{x 1}\right)$ to compensate the poor resolution of RSSI in Wi-Fi device, which will decrease the performance of localization algorithm when the APs' distance increase. As shown in Fig. 10, when Dist $=25 \mathrm{~m}$, the localization algorithm performs worse than that of Dist $=20 \mathrm{~m}$ with enhanced resample/weighted algorithm, and achieves a performance more or less the same with the configuration of Dist=20m and without enhanced algorithm. DWELT algorithm shows better performance when the distance between two adjacent APs is within a reasonable value (around 20 meters). The localization errors tend to be higher as the distance increases. Besides, the huge obstacles in the line-of-sight of APs will affect the localization accuracy significantly. 


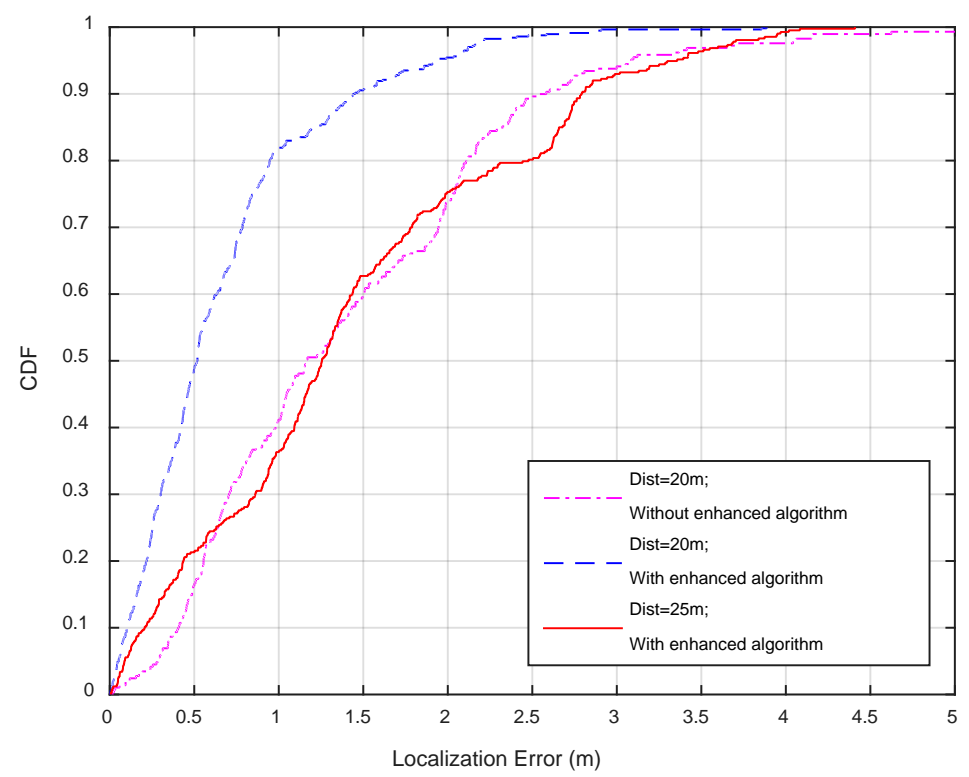

Fig. 10. CDF of localization error with/without enhanced resample/weighted algorithm

\section{Conclusions and future work}

In this paper, we propose and evaluate the Dynamic Weighted Evolution for Location Tracking (DWELT), an enhanced RSSI-based positioning algorithm intended to provide accurate indoor positioning of Wi-Fi-based sensors, which leverages the ubiquity of Wi-Fi infrastructure in many of such scenarios. DWELT is simple: it does not need any extra signaling or cooperation among Wi-Fi APs, and it does not require a prior data harvesting phase (e.g. to develop a fingerprint database). DWELT is fast: it shows low computational overhead (implemented and tested on Android-based smartphones). DWELT is accurate: simulations as well as experimental measurements showed that DWELT provides location within $1 \mathrm{~m}$ precision in more than $81 \%$ of the cases, and within $2 m$ in $95 \%$.

\section{Acknowledgement}

This work is supported in part by the National Natural Science Foundation (NSF) of China (Grant No. 61571330); the Harman-Tongji Joint Research Project (Grant No. 08002450266); the Spanish Government's Ministerio de Economía y Competitividad through project TEC2012-32531, and by the FEDER. 


\section{References}

[1] M. A. Al-Ammar, S. Alhadhrami, A. Al-Salman, A. Alarifi, H. S. Al-Khalifa, A. Alnafessah, and M. Alsaleh, "Comparative Survey of Indoor Positioning Technologies, Techniques, and Algorithms," in Proc. of International Conference on Cyberworlds, pp. 245-252, Oct., 2014. Article (CrossRef Link).

[2] Wi-Fi Alliance, “Total Wi-Fi device shipments to surpass ten billion this month,” Jan., 2015. Article (CrossRef Link).

[3] C. Yang, H. R. Shao, “WiFi-based indoor positioning,” IEEE Communications Magazine, vol. 53, no. 3, pp. 150-157, Mar., 2015. Article (CrossRef Link).

[4] I. Bisio, M. Cerruti, and et al., "A trainingless wifi fingerprint positioning approach over mobile devices," IEEE Antennas and Wireless Propagation Letters, vol.13, pp. 832-835, 2014. Article (CrossRef Link).

[5] J. Tang, Y. Chen, L. Chen, J. Liu, J. Hyyppä, A. Kukko, H. Kaartinen, H. Hyyppä, and R. Chen, "Fast Fingerprint Database Maintenance for Indoor Positioning Based on UGV SLAM,” Sensors, vol. 15, no. 3, pp. 5311-5330, Mar., 2015. Article (CrossRef Link).

[6] Y. Du, D. Yang, C. Xiu, "A Novel Method for Constructing a WIFI Positioning System with Efficient Manpower,” Sensors, vol. 15, no. 4, pp. 8358-8381, Apr., 2015. Article (CrossRef Link).

[7] Y. Mo, Z. Zhang, Y. Lu, and et al., "A Novel Technique for Human Traffic based Radio Map Updating in Wi-Fi Indoor Positioning Systems," KSII Transactions on Internet and Information Systems (TIIS), vol. 9, no. 5, pp. 1881-1903, 2015. Article (CrossRef Link).

[8] J. Huang, D. Millman, and et al., "Efficient, generalized indoor wifi graphslam," in Proc. of Robotics and Automation (ICRA), 2011 IEEE International Conference on., pp. 1038-1043, Sept., 2011. Article (CrossRef Link).

[9] L. Bruno, P. Robertson, "Wislam: Improving footslam with wifi," in Proc. of Indoor Positioning and Indoor Navigation (IPIN), 2011 International Conference on., IEEE, pp. 1-10, May, 2011. Article (CrossRef Link).

[10] H. Wang, S. Sen, and et al., "No need to war-drive: unsupervised indoor localization," in Proc. of the 10th international conference on Mobile systems, applications, and services, pp. 197-210, 2012. Article (CrossRef Link).

[11] C. H. Lim, Y. Wan, and et al., "A real-time indoor WiFi localization system utilizing smart antennas," IEEE Transactions on Consumer Electronics, vol. 53, no. 2, pp. 618-622, May, 2007. Article (CrossRef Link).

[12] D. J. Suroso, P. Cherntanomwong, P. Sooraksa, and J. Takada, "Fingerprint-based technique for indoor localization in wireless sensor networks using Fuzzy C-Means clustering algorithm," in Proc. of 2011 International Symposium on Intelligent Signal Processing and Communications Systems (ISPACS), pp. 1-5, Dec., 2011. Article (CrossRef Link).

[13] P. Sunantasaengtong and S. Chivapreecha, "Mixed K-means and GA-based weighted distance fingerprint algorithm for indoor localization system," in Proc. of TENCON 2014-2014 IEEE Region 10 Conference, pp. 1-5, Oct., 2014. Article (CrossRef Link).

[14] M. Shon, M. Jo, and et al., "An interactive cluster-based MDS localization scheme for multimedia information in wireless sensor networks," Computer communications, vol. 35, no. 15, pp. 1921-1929, Sept., 2012. Article (CrossRef Link).

[15] L. Gogolak, S. Pletl, and D. Kukolj, "Indoor fingerprint localization in WSN environment based on neural network," in Proc. of 2011 IEEE 9th International Symposium on Intelligent Systems and Informatics, pp. 293-296, Sept., 2011. Article (CrossRef Link). 
[16] Z. Chen, H. Zou, H. Jiang, Q. Soh, and et al., "Fusion of WiFi, Smartphone Sensors and Landmarks Using the Kalman Filter for Indoor Localization,” Sensors, vol. 15, no. 1, pp. 715-732, Jan., 2015. Article (CrossRef Link).

[17] D. Sánchez-Rodríguez, P. Hernández-Morera, J. M. Quinteiro, I. Alonso-González, "A Low Complexity System Based on Multiple Weighted Decision Trees for Indoor Localization,” Sensors, vol. 15, no. 6, pp. 14809-14829, Jun., 2015. Article (CrossRef Link).

[18] A. Patri and S. P. Rath, "Elimination of Gaussian noise using entropy function for a RSSI based localization," in Proc. of 2013 IEEE Second International Conference on Image Information Processing (ICIIP-2013), pp. 690-694, Dec., 2013. Article (CrossRef Link).

[19] C. H. Huang, L. H. Lee, and et al., "Real-Time RFID Indoor Positioning System Based on Kalman-Filter Drift Removal and Heron-Bilateration Location Estimation," IEEE Transactions on Instrumentation and Measurement, vol. 64, no. 3, pp. 728-739, Mar., 2015. Article (CrossRef Link).

[20] B. Solhjoo and M. A. Tinati, "An adaptive environmental modeling localization method in Wireless Sensor Networks," in Proc. of 20th Iranian Conference on Electrical Engineering (ICEE2012), pp.1397-1402, May, 2012. Article (CrossRef Link).

[21] Y. Guo, K. Huang, N. Jiang, X. Guo, Y. Li, and G. Wang, “An Exponential-Rayleigh Model for RSS-Based Device-Free Localization and Tracking," IEEE Trans. on Mobile Comput., vol. 14, no. 3, pp. 484-494, Mar., 2015. Article (CrossRef Link).

[22] C. B. Lim, S. H. Kang, H. H. Cho, S. W. Park, and J. G. Park, "An Enhanced Indoor Localization Algorithm Based on IEEE 802.11 WLAN Using RSSI and Multiple Parameters," in Proc. of 2010 Fifth International Conference on Systems and Networks Communications, pp. 238-242, Aug., 2010. Article (CrossRef Link).

[23] J. S. Seybold, “Introduction to RF propagation,” John Wiley \& Sons, 2005. Article (CrossRef Link).

[24] A. Bose, C. H. Foh, "A practical path loss model for indoor WiFi positioning enhancement," in Proc. of Information, Communications \& Signal Processing, 2007 6th International Conference on., pp. 1-5, Dec., 2007. Article (CrossRef Link).

[25] H. Ni, W. Xu, Y. Li, M. Tao, S. Song, H. Fan, "An Improved Method of Self-Adaptive Localization for Wireless Sensor Network in Dynamic Indoor Environment,” in Proc. of 31st Chinese Control Conference (CCC), pp.6574-6577, Jul., 2012. Article (CrossRef Link).

[26] W. Daamen, S. P. Hoogendoorn, "Free speed distributions for pedestrian traffic," in Proc. of the 85th Annual Meeting of Transportation Research Board, pp. 22-26, 2006. Article (CrossRef Link).

[27] E. C. Chan, G. Baciu, S. C. Mak, "Using Wi-Fi signal strength to localize in wireless sensor networks," in Proc. of Communications and Mobile Computing, CMC'09. WRI International Conference on., IEEE, vol. 1, pp. 538-542, Jun., 2009. Article (CrossRef Link).

[28] V. Erceg, “TGn channels models,” IEEE P802.11TGn, doc IEEE 802.11-03/0940r4., 2004. Article (CrossRef Link). 

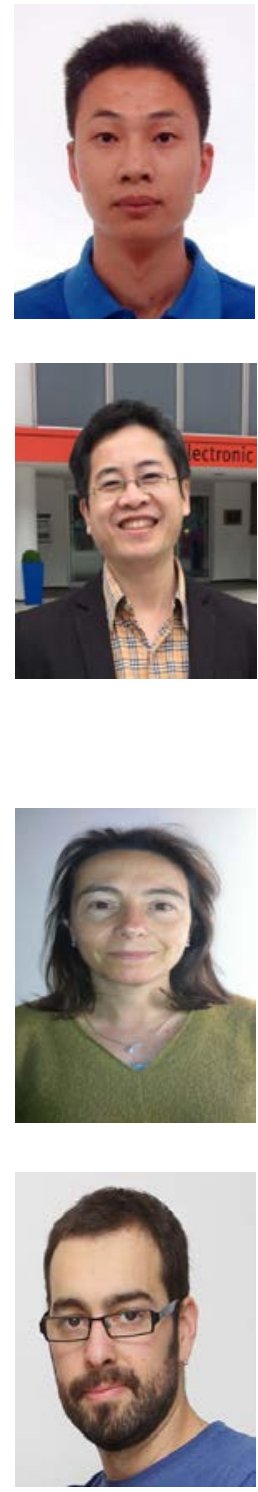

Weideng Su received his B.E. degree in Communication Engineering from Tongji University, P. R. China, in June 2015. He is currently pursuing a master's degree at the College of Electronics and Information Engineering in Tongji University. His current research interests include indoor localization and wireless sensor networks.

Erwu Liu obtained his Ph.D. in 2001, from the state key laboratory in Huazhong University of Science and Technology. In 2001-2007, he served Alcatel-Lucent as project manager, senior consultant, and senior research scientist. From 2007 to 2011, he was with Imperial College London, working as a research associate. Since 2011, he has been a professor in Tongji University. Professor Liu is an associate editor of the IEEE Communications Letters, and an editor of the KSII Transactions on Internet and Information and Systems. Currently, he is a fellow of the IET, ALTA member of Alcatel-Lucent technical academy, and senior members of both ACM and IEEE. His research interests include complex network theory and big data, wireless sensor networks, deep-penetration communications, indoor localization, and 5G technologies.

Dr. Anna Calveras Augé obtained the Ph.D. degree from the Universitat Politècnica de Catalunya in 2000. She is associate professor at the mentioned University, at the Telematics Department, in the Wireless Networks Group (WNG). Her research interests and expertise areas comprise the design, evaluation and optimization of communications protocols for wireless multihop networks, ad-hoc networks, wireless sensor networks, the Internet of Things, and application domains such as smart cities and building automation. She has been involved in several National and International research or technology transfer projects, and she has published in International and National conferences and journals.

Dr. Eduard Garcia-Villegas received his MSc and $\mathrm{PhD}$ degrees from the Technical University of Catalonia, Barcelona Tech (UPC) in 2003 and 2010, respectively. He is associate professor in the same university and a member of the Wireless Networks Group (WNG). He participates in the activities of the IEEE P802.11 WG as a voting member. He also participates in the research developed within the i2CAT Foundation and, after being awarded a José Castillejo Grant for young doctors, he occasionally collaborates with the MOMENT Lab at UC Santa Barbara. His research interests include topics such as radio resource optimization of WLANs and WPANs, cross layer optimization, network security, and Internet of Things (IoT) enabling technologies (sensor networks, mesh, multi-hop ad-hoc networks, etc.). 


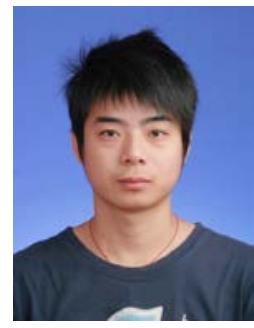

Rui Wang (M'14) received the Ph.D. degree from Shanghai Jiao Tong University, China, in 2013, in electronic engineering. From Aug. 2012 to Feb. of 2013, he was a visiting Ph.D student at the Department of Electrical Engineering of University of California, Riverside. From Oct. 2013 to Oct. of 2014, he was with the Institute of Network Coding, The Chinese University of Hong Kong as a postdoctoral research associate. Since Oct. 2014, he has been with the College of Electronics and Information Engineering, Tongji University as an assistant professor. His research interests include wireless cooperative communications, MIMO technique, network coding, and OFDM etc.

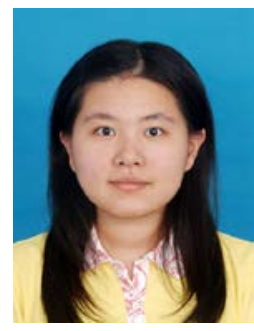

Jiayi You received her B.E. degree in Communication Engineering from East China Normal University, P. R. China, in June 2015. She is currently pursuing a master's degree at the College of Electronics and Information Engineering in Tongji University. Her current research interests include wireless cooperative communications, MIMO technique and indoor positioning etc. 